\title{
Solar radiative transfer simulations in Saharan dust plumes: particle shapes and 3-D effect
}

\author{
By ANTJE TORGE ${ }^{1,2 *}$, ANDREAS MACKE ${ }^{2}$, BERND HEINOLD ${ }^{2}$ and JOCHEN WAUER ${ }^{3}$, \\ ${ }^{1}$ Leibniz-Institut für Meereswissenschaften (IFM-GEOMAR), Düsternbrooker Weg 20, 24105 Kiel, Germany; \\ ${ }^{2}$ IFT, Leipzig, Germany; ${ }^{3}$ University of Leipzig, Institute for Meteorology, Germany
}

(Manuscript received 2 November 2010; in final form 31 May 2011)

\begin{abstract}
Radiative fields of three-dimensional inhomogeneous Saharan dust clouds have been calculated at solar wavelength $(0.6 \mu \mathrm{m})$ by means of a Monte Carlo radiative transfer model. Scattering properties are taken from measurements in the SAMUM campaigns, from light scattering calculations for spheroids based on the MIESCHKA code, from Mie theory for spheres and from the geometric optics method assuming irregular shaped particles. Optical properties of different projected area equivalent shapes are compared. Large differences in optical properties are found especially in the phase functions.

Results of radiative transfer calculations based on the Monte Carlo method are shown exemplarily for one dust cloud simulated by the cloud resolving atmospheric circulation model LM-MUSCAT-DES. Shape-induced differences in the radiation fluxes are pronounced, for example, the domain averaged normalized radiance is about $30 \%$ lower in the case of a dust plume consisting of spheroids or irregular particles compared to spheres. The effect of net horizontal photon transport (3-D effect) on the reflected radiance fields is only notable at the largest gradients in optical thickness. For example, the reflectance at low sun position differs locally about $15 \%$ when horizontal photon transport is accounted for. 'Sharp edges' due to 1-D calculations are smoothed out in the 3-D case.
\end{abstract}

\section{Introduction}

Estimates of the amount of global dust aerosol vary between 1000 and $5000 \mathrm{Tg} \mathrm{yr}^{-1}$. Therefore, dust in the atmosphere plays an important role in the climate system. The Saharan desert, especially with the Bodélé Depression is the world's largest source region of atmospheric mineral dust. About $61000 \mathrm{~km}^{3}$ diatomite has been eroded from Bodélé during the past $1000 \mathrm{yr}$ (Bristow et al., 2009).

The anthropogenic part of global dust load increases due to land use practices and construction. The energy distribution in the atmosphere, the global radiation budget (Tegen et al., 1996), the cloud life cycle (Kaufman et al., 2005) and the formation of tropical cyclones (Evan et al., 2006) are influenced by dust aerosol.

Dust in the troposphere also has a direct effect on animals, vegetation and human health. The dust from Africa carries viable micro-organisms, macro and micro nutrients, trace metals and some organic contaminants. Deposited in the ocean and on land, dust may play an important role in the complex changes in some

\footnotetext{
* Corresponding author.

e-mail: atorge@ifm-geomar.de

DOI: $10.1111 / \mathrm{j} .1600-0889.2011 .00560 . \mathrm{x}$
}

ecosystems, for example, in coral reefs worldwide (Griffin et al., 2001; Garrison et al., 2003).

The mineral dust acts as a fertilizer in the Amazon rain forest as well as the equatorial Atlantic Ocean and influences, by that, indirectly the $\mathrm{CO}_{2}$ cycle (Bristow et al., 2010).

The dimension of dust effects depends on the particle chemical, physical and optical properties which differ with the source region. The airborne dust itself is mainly influenced by wind fields and precipitation (Schepanski et al., 2007).

The Saharan Mineral Dust Experiment (SAMUM) investigates the properties of the dust particles and quantifies dustrelated radiative effects. The field campaign of the first phase took place near a dust source region in Southern Morocco 2006 and continued with the second phase far field studies at Cape Verde Islands in 2008. In the campaigns two research aircrafts, one equipped with a lidar, and three ground-based lidars have been used. The detailed observations were applied to a dust transport model and to radiative transfer models whose results were compared to satellite measurements (Heintzenberg, 2009; Ansmann et al., 2011).

This study is focused on radiative transfer simulations in Saharan dust plumes in the framework of SAMUM with special attention to different particle shapes and horizontal photon transport. 
Scattering properties of spherical, spheroidal and irregular shaped particles are compared. There are several other studies considering different shapes in radiative transfer simulations in dust plumes, but the uncertainties in the particle size distribution of the dust cloud and its refractive index, which is very sensitive to particle size and chemical composition depending on the source region, make it difficult to compare the results. The studies by, for example, Otto et al. (2009), Yang et al. (2007), Kalashnikova and Sokolik (2004) and Fu et al. (2009) show that the differences in scattering properties between spherical and spheroidal particles are small as it is confirmed in this study. In Kalashnikova and Sokolik (2002) and Kalashnikova and Sokolik (2004) other particle shapes and mixtures of a variety of shapes are compared. They found larger differences in the scattering properties compared to spherical particles.

3-D radiative effects due to horizontal photon transport have been studied mostly for water, mixed phase and ice clouds, for example, by Scheirer and Macke (2003), Benner and Evans (2001), Davis et al. (1997) and Di Giuseppe and Tompkins (2003) among others.

The aim of this study is to extend these investigations to 3-D mineral dust clouds with a special emphasis on the non-spherical dust particle geometries. In this regard, dust clouds pose a similar challenge to radiative transfer investigations like cirrus clouds (Schlimme et al., 2005).

\section{Models and methods}

\subsection{LM-MUSCAT-DES}

LM-MUSCAT-DES is a system of regional models to simulate emission, transport, deposition and radiative effects of Saharan dust in northern Africa and parts of the Atlantic Ocean. The Dust Emission Scheme (DES) was developed by Tegen et al. (2002).
Surface properties like roughness, soil size distribution, vegetation cover, soil moisture content and the location of preferential dust sources are considered to calculate the erosion threshold velocity and horizontal and vertical dust fluxes. These are used in the multiscale chemistry-transport code (MUSCAT) to simulate the transport and deposition of dust in the framework of the SAMUM campaign. MUSCAT is coupled online with the non-hydrostatic meteorological model (LM). It is operated with initial and boundary conditions from the global model GME of the German Weather Service, DWD. Originally, MUSCAT was developed to simulate the dynamics of pollutants, so DES and dry and wet deposition were embedded. There are modules included which describe aerosol dynamics and chemical mechanisms. The dust is transported as a dynamic tracer in five independent size classes (from bin1 to bin5) whose radius limits are $0.1 \mu \mathrm{m}, 0.3 \mu \mathrm{m}, 0.9 \mu \mathrm{m}, 2.6 \mu \mathrm{m}, 8 \mu \mathrm{m}$ and $24 \mu \mathrm{m}$ (Heinold et al., 2007).

The LM-MUSCAT-DES was validated with measurements of optical thicknesses, extinction coefficients and particle size distributions as well as satellite dust indices and standard meteorological parameters during the SAMUM field campaign. Dust optical properties and size distributions are mostly well matched by the model. However, the spatio-temporal evolution of the dust plumes was not always satisfactorily reproduced (Heinold et al., 2007).

In Fig. 1, a qualitative comparison of modelled and satellite measured spatial dust cloud pattern for 31 January 2008 13:00 UTC is shown. Figure 1(a) shows the amount of particles of the MUSCAT modelled cloud in pink and the green shaded surface albedo. As surface albedo was used a $0.3-0.5 \mu \mathrm{m}$ broad-band inhomogeneous surface albedo field from MODIS with the same resolution as the MUSCAT model. In (b) the corresponding IR Index of the same date and area can be seen. The IR Index (http://www.eumetsat.int) is only a qualitative index for the
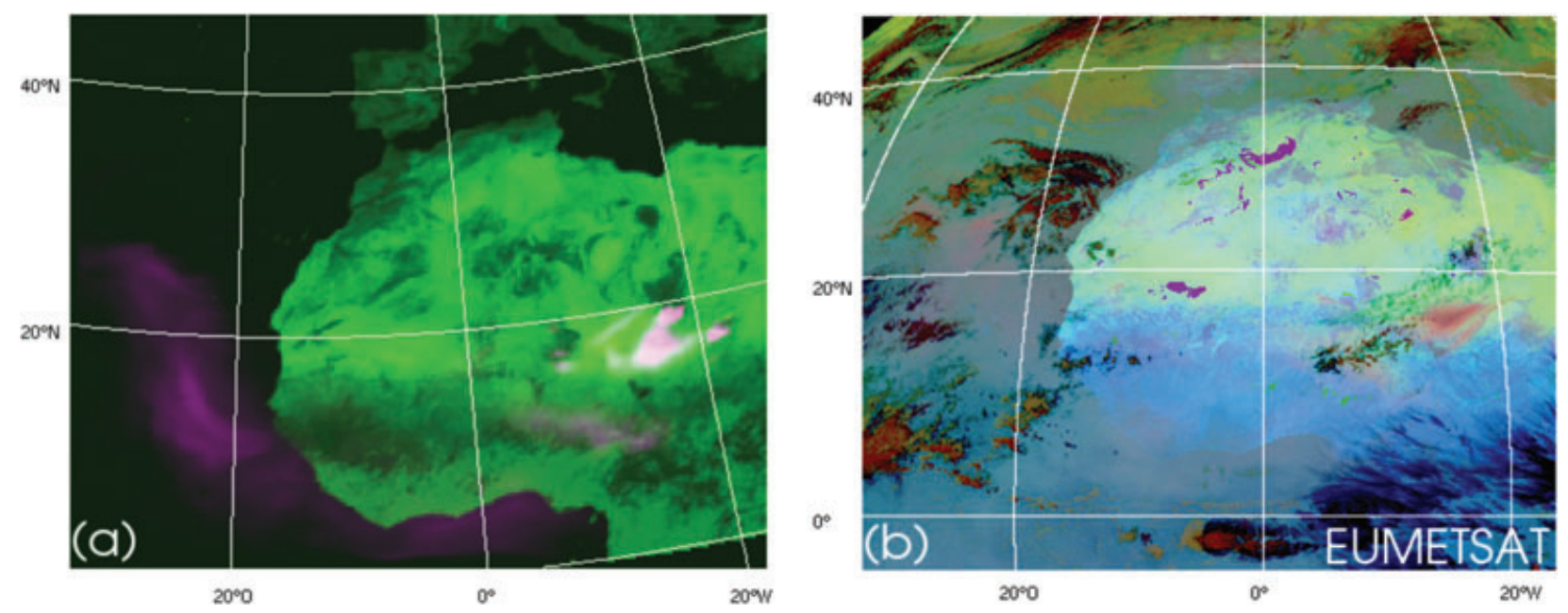

Fig. 1. Dust cloud from 31 January 2008 13:00 UTC (a) MUSCAT modelled dust cloud in pink with green shaded surface albedo (b) IR Index from MSG satellite measurements, red to pink colour indicates dust. 
occurrence of dust. It is a false colour image composite based on brightness temperature differences measured by the infrared channels of the SEVIRI instrument onboard of the geostationary MSG satellite which is placed in $0^{\circ}$ longitude and has a resolution of $3 \mathrm{~km}$. The IR Index indicates dust with red to magenta colours (Wald et al., 1998). Both pictures show nearly the same patterns and locations of the dust plumes. There are light red dusty shades west of North Africa over the Atlantic and a clearly visible dust cloud between Lake Chad and the Bodélé Depression. Another lighter dusty area is located south west of the larger one. In general the observed and modelled dust cloud pattern are in good agreement. In the following calculations only the smaller part with the clear visible dust cloud between Lake Chad and the Bodélé Depression was used because of computation limitations.

\section{2. $M C-U n i K$}

The Monte Carlo Model of the University of Kiel (MC-UniK, Macke et al., 1999) is a forward model for efficient calculations of radiances at discrete directions. It includes the 'Local Estimate Method' (Marshak and Davis, 2005) and it was validated within the I3RC project (Intercomparison of 3-D Radiation Codes (http://i3rc.gsfc.nasa.gov/, Cahalan et al., 2005).

MC-UniK simulates directly the path of photons through the dust cloud. Accuracy increases with the number of photons. The free path length is based on Beers Law and gives the step size between two successive scattering processes. A random process weighted with the scattering phase function results in the scattering angle. Absorption is taken into account with the photon weight multiplied by the local single scattering albedo.

For calculating the radiance field the 'Local Estimate Method' is more efficient than the common Monte Carlo photon counting method, because no photons get lost. Additional to each scattering process the part of the local photon energy which is directly scattered along the path towards the detector is considered. This part is attenuated at the length of the way to the detector and according to the probability of scattering into that direction registered.

The model domain is separated into grid boxes which are characterized by their optical properties. The input data required for MC-UniK for each model grid box are the coordinates in $x, y$ and $z$ direction, the extinction coefficient $\beta_{\text {ext }}$, the single scattering albedo $\omega_{0}$ and the scattering phase function $P(\theta)$ depending on the scattering angle $\theta$. Furthermore, the sun position, observer position, number of photons and surface albedo are required.

The illustration in Fig. 2 visualizes the general performance of the Monte Carlo method. The photons from the sun direction reach the 3-D Cartesian cuboidal model domain. Different colours symbolize different optical properties of the dust cloud grid boxes. Photons are transmitted, absorbed and scattered on their way through the domain. The dotted lines symbolize the

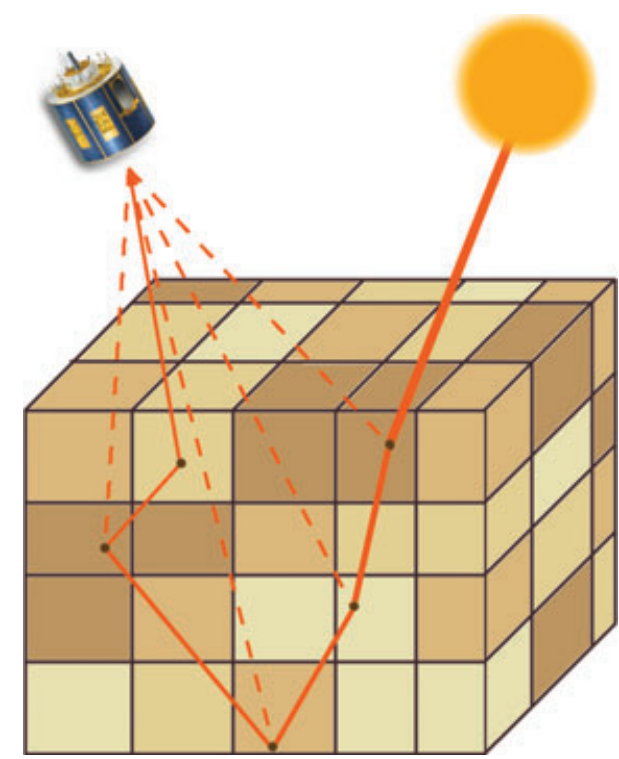

Fig. 2. Schematic way of photons from sun through a cubic dust cloud to the observing MSG satellite with different optical properties in each grid box. Dotted lines symbolize 'Local Estimate Method'.

'Local Estimate Method' considering the part of photons directly scattered into the satellite's viewing angle at each scattering process.

To perform the MC-UniK calculations, the given size distribution from a MUSCAT cloud is converted into optical properties based on the scattering classes (Section 2.3) assuming spherical, spheroidal and irregular shaped dust particles. The results from the MC-UniK calculation with spherical particles for the chosen part of the dust cloud from 31 January 2008 13:00 UTC plotted in Fig. 3(a) show the amount of particles of the MUSCAT modelled cloud and (b) the corresponding IR Index from the MSG measurements. The IR Index indicates the dust in pink to red colours.

The simulated normalized radiances from MC-UniK and the measured radiance from the satellite (channel 1 of the SEVIRI instrument) are illustrated in (c) and (d).

\subsection{Scattering classes}

Since the modelled domain from the LM-MUSCAT-DES consists of $182 \times 229$ horizontal boxes and 40 vertical layers, there are over 1.5 million dust cloud boxes. For each box the scattering properties including the scattering phase functions are required. To manage this amount of data, the size- and shape-dependent scattering properties have been discretized into 14 classes and their assumed shape as shown in Table 2.

The ratio between small and large particles is an appropriate measure for the scattering properties of one grid box with a given discrete size distribution. First the boxes were grouped into five classes depending in which of the five size classes the 

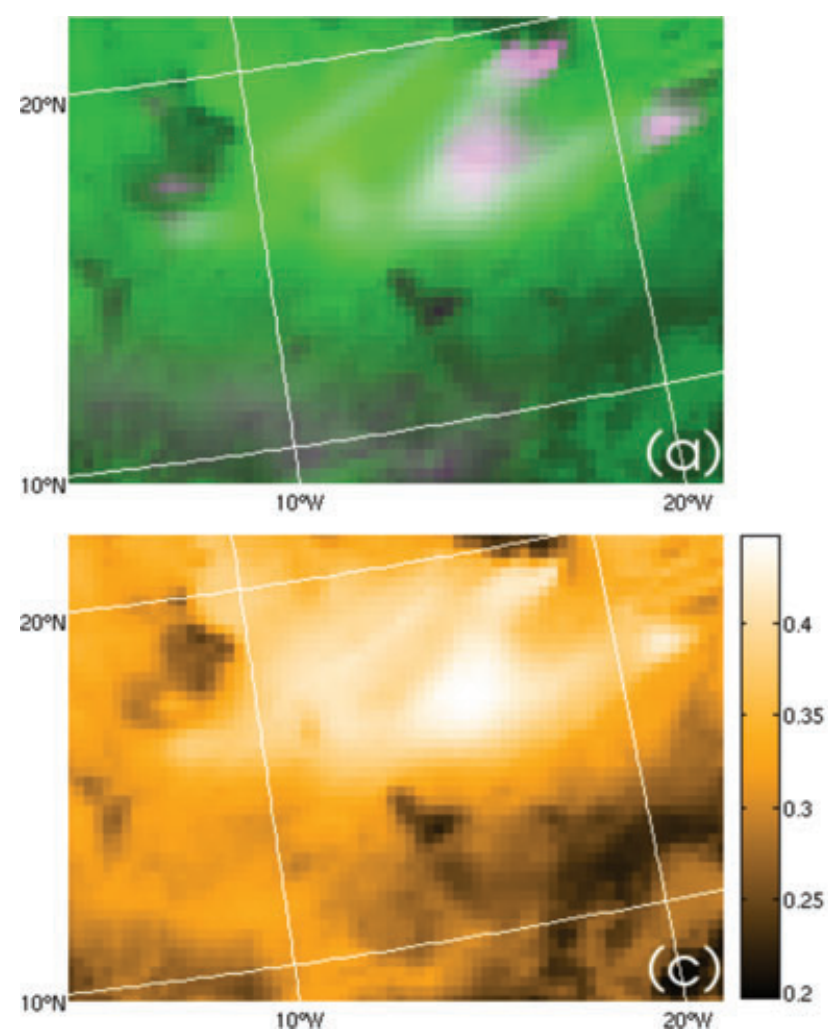
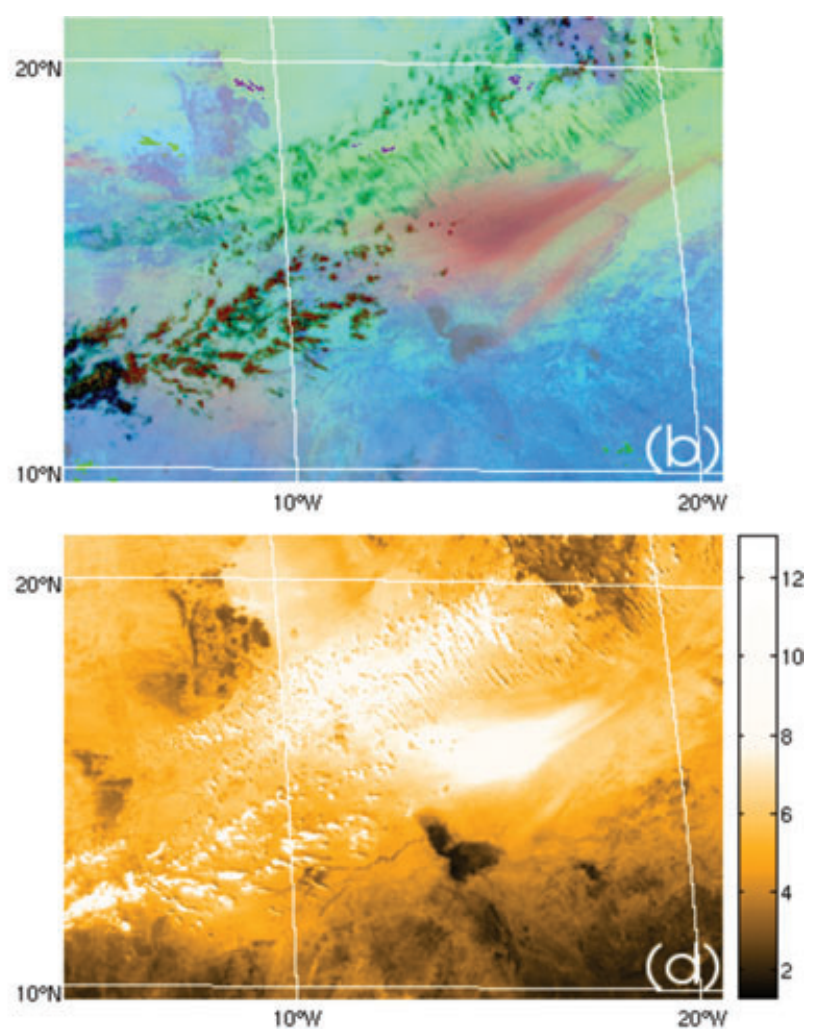

Fig. 3. The Monte Carlo simulated part of the dust Cloud from 31 January 2008 13:00 UTC. (a) MUSCAT cloud, (b) IR Index from the MSG satellite, (c) MC-UniK simulated normalized radiance for spherical particles at $0.6 \mu \mathrm{m}$ and (d) measured radiance from MSG around the $0.6 \mu \mathrm{m}$ channel in $\mathrm{W} \mathrm{m}^{-2} \mathrm{sr}^{-1}$; colour bar is a little shifted because of the highly reflecting water clouds which do not occur in the model.

largest number of particles is located. Because in most cases the number of the smallest particles (bin1 with effective radius $0.166 \mu \mathrm{m}$ ) is the largest, this class is split up into 10 additional classes depending on the ratio between small and large particles as shown in Table 2. For the 14 classes the averaged optical properties of the classified boxes were taken. Sensitivity studies with undiscretized 3-D variable scattering and absorption properties (not shown here) have shown that the discretization errors are small compared to the Monte Carlo noise.

Figure 4 shows the discretized extinction efficiency and single scattering albedo for spherical particles. The values with black dots are calculated with Mie Theory for all dust cloud boxes and the grey circles show the chosen average values for the corresponding classes. The discretization is assumed to sufficiently represent the microphysical and thus optical variability in the 3 -D radiative transfer calculations.

\section{Monte Carlo calculations}

\subsection{Dust scattering properties for different shapes}

To study the role of different particle shapes and their influence on the simulated radiation fields, the scattering properties of spheres, ellipsoids and irregular shaped particles have been compared. The different shaped particles were taken in the way that the amount of particles in the cloud did not change and all particles have nearly the same surface. Thus the volume of spheroidal particles is $3 \%$ smaller than that of spheres. Mie Theory is used for spherical particles.

The Scattering Database for spheroidal particles (Schmidt et al., 2009) provides scattering properties for a wide range of different particles. However, for best agreement with the measured data from the SAMUM Campaign, scattering at spheroidal particles has been calculated with the code MIESCHKA (Wauer et al., 2004) via the Virtual Lab (http://vl.nz.dlr.de/VL) environment. MIESCHKA is based on a generalization of the separation of variables method and can be used for particles up to a size parameter of 40 . For larger spheroids, as in the case of the two largest size classes bin4 (size parameter 47.4, effective radius $4.571 \mu \mathrm{m}$ ) and bin5 (size parameter 143.18, effective radius $13.804 \mu \mathrm{m}$ ), a ray tracing code (Macke et al., 1997) based on the geometric optics method was used. The methods used to calculate the scattering properties for different shapes and particle sizes are summarized in Table 1 .

An aspect ratio of 1.6 and a refractive index of $1.574+0.0011 \mathrm{i}$ at a wavelength of $0.6 \mu \mathrm{m}$ for dust particles according to the measurements in the SAMUM field campaigns from Kandler et al. (2009) and Kandler et al. (2011) are assumed. 

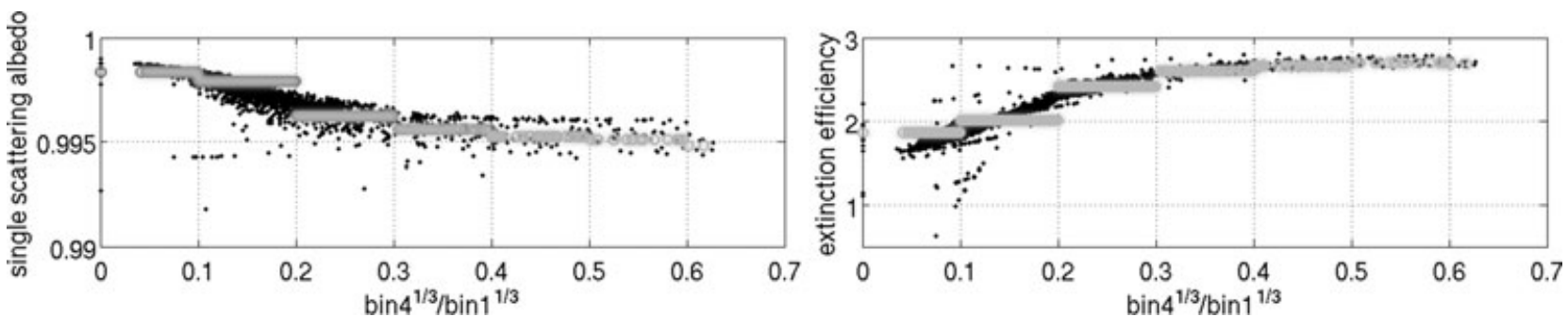

Fig. 4. Extinction efficiency and single scattering albedo at $1.6 \mu \mathrm{m}$ calculated exact for each cloud box with Mie theory (black dots) and the discretized values in grey circles.

Table 1. Used methods to calculate scattering properties for different shapes and particle sizes (extinction efficiency for ray tracing method was always assumed to be the same as for the corresponding sphere calculated with Mie Theory)

\begin{tabular}{|c|c|c|c|c|c|c|}
\hline Shape & $\begin{array}{c}\text { bin } 1 \\
0.166 \mu \mathrm{m}\end{array}$ & $\begin{array}{c}\operatorname{bin} 2 \\
0.501 \mu \mathrm{m}\end{array}$ & $\begin{array}{c}\operatorname{bin} 3 \\
1.514 \mu \mathrm{m}\end{array}$ & $\begin{array}{c}\text { bin4 } \\
4.571 \mu \mathrm{m}\end{array}$ & $\begin{array}{c}\text { bin5 } \\
13.804 \mu \mathrm{m}\end{array}$ & Aspect ratio \\
\hline Sphere & \multicolumn{5}{|c|}{ Mie Theory } & 1 \\
\hline Spheroid & \multicolumn{3}{|c|}{ MISCHKA } & \multicolumn{2}{|c|}{ Ray tracing } & 1.6 \\
\hline Irregular particle & Mie Theory & \multicolumn{4}{|c|}{ Ray tracing } & 1.6 \\
\hline
\end{tabular}

However, one should keep in mind that the scattering properties of dust particles are very sensitive to the refractive index and even small uncertainties on this value can have big influences on the scattering properties and thus on the calculated radiance fields. To quantify these influences more calculations with different refractive indices have to be performed, which, however, is beyond the scope of this study.

The ray tracing method was also used for calculating the scattering properties of irregular shaped particles that are considerably larger than the wavelength. These irregular particles (Fig. 5) are randomly constructed convex polyhedrons with triangular surfaces and an aspect ratio of about 1.6.

For smaller irregular particles as in the size class bin 1 the scattering properties were assumed to be identical to those from spherical ones. This assumption results in smaller differences in the size distribution averaged scattering properties of distinct shapes for the scattering classes.

In the upper part of Fig. 6 the single scattering albedo and the extinction efficiency for the five size classes from the MUSCAT model are plotted. Only small differences can be seen for the single scattering albedo. Because of limitations of the geometric optics method approximation, the irregular particle extinction efficiency is always 2 and was replaced by the value for the projected area equivalent sphere. There are significant differences between spheres and spheroids. The extinction efficiencies of spheroids differ up to 0.5 in the size class bin2 (effective radius $0.501 \mu \mathrm{m}$ ) from the ones of spheres. Also large shape-induced differences are obvious in the scattering phase functions, displayed in Fig. 6. All shape-induced differences in scattering properties increase with particle size.

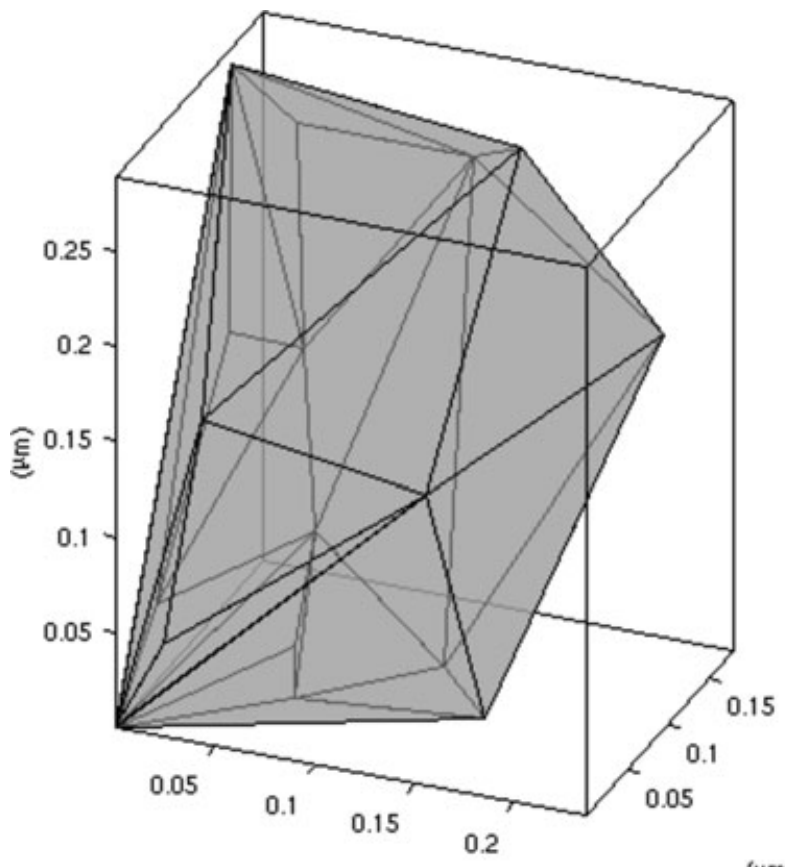

$(\mu \mathrm{m})$

$(\mu \mathrm{m})$

Fig. 5. Example of irregular shaped particle with triangular surfaces.

Each of the 14 scattering classes is a combination of the five size classes defined earlier. So the optical properties of the scattering classes are weighted averages of the optical properties of the size classes. The composition is shown in Table 2 .

Figure 7 and Table 3 show the scattering properties of the 14 scattering classes. The single scattering albedo and the extinction 

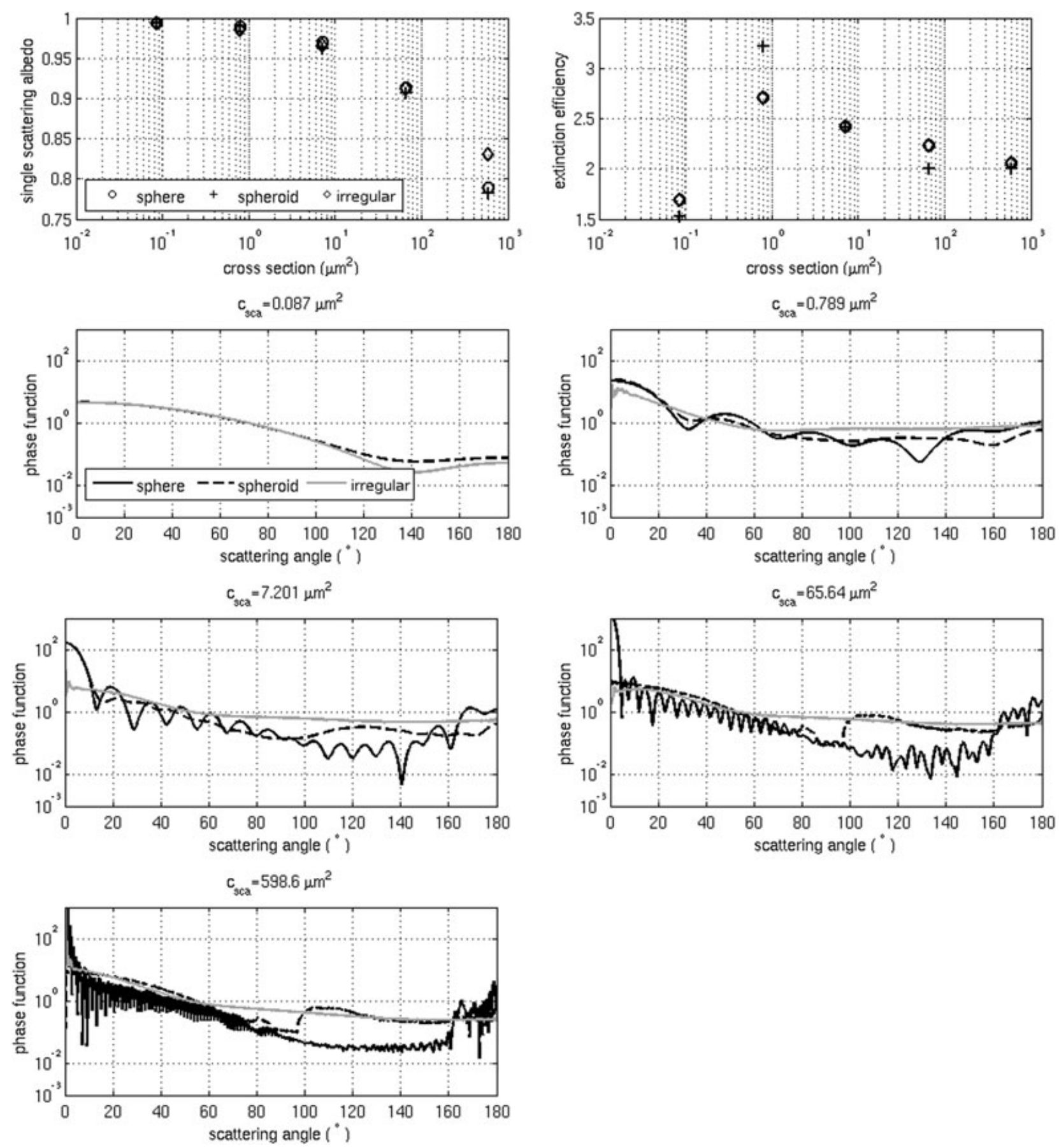

Fig. 6. Extinction efficiency, single scattering albedo and scattering phase functions for five particle size classes with their cross section for three different shapes.

efficiency depend on the effective radius of the scattering class. There are only small differences in the single scattering albedo for the three particle shapes. The values for spheroids and irregular particles differ less than $1 \%$ from the single scattering albedo of spheres.

In the study by Otto et al. (2009), which deals with radiative transfer calculations for dust clouds consisting of spherical and

spheroidal dust particles, it is also shown that at a wavelength of $0.6 \mu \mathrm{m}$ the differences between spheroids and spheres are small. The single scattering albedo is about $0.5 \%$ lower for spheroids than for spheres, but the values reported by Otto et al. (2009) are smaller in general. This may mainly be caused by different input data for particle size distributions and refractive index due to measured data at different time and location. 
Table 2. Development and composition of the 14 scattering classes as described in Section 2.3 'Scattering classes'. Bin1 to bin5 are 5 size classes with corresponding effective radius

\begin{tabular}{|c|c|c|c|c|c|c|c|c|c|}
\hline \multirow{2}{*}{$\begin{array}{c}\text { Size bin class } \\
\text { with biggest } \\
\text { particle } \\
\text { amount }\end{array}$} & \multirow{2}{*}{$\begin{array}{c}\text { Ratio between } \\
\text { big and small } \\
\text { particles }\end{array}$} & \multirow{2}{*}{$\begin{array}{l}\text { Occurrence of } \\
\text { scattering class } \\
\text { in dust cloud } \\
\text { boxes in \% }\end{array}$} & \multicolumn{5}{|c|}{ Composition of the scattering classes in $\%$} & \multirow{2}{*}{$\begin{array}{c}\text { Effective } \\
\text { radius } \\
(\mu \mathrm{m})\end{array}$} & \multirow[b]{2}{*}{ Name } \\
\hline & & & $\begin{array}{c}\text { bin } 1 \\
0.166 \mu \mathrm{m}\end{array}$ & $\begin{array}{c}\text { bin } 2 \\
0.501 \mu \mathrm{m}\end{array}$ & $\begin{array}{c}\text { bin } 3 \\
1.514 \mu \mathrm{m}\end{array}$ & $\begin{array}{c}\text { bin } 4 \\
4.571 \mu \mathrm{m}\end{array}$ & $\begin{array}{c}\text { bin } 5 \\
13.804 \mu \mathrm{m}\end{array}$ & & \\
\hline \multirow[t]{10}{*}{ bin 1} & $0.0-0.1$ & 14.31 & 61.82 & 36.10 & 2.03 & 0.05 & 0.00 & 2.098 & 1.1 \\
\hline & $0.1-0.2$ & 67.38 & 60.85 & 36.53 & 2.47 & 0.14 & 0.00 & 2.866 & 1.2 \\
\hline & $0.2-0.3$ & 6.97 & 56.92 & 37.02 & 5.24 & 0.81 & 0.01 & 5.887 & 1.3 \\
\hline & $0.3-0.4$ & 1.89 & 52.38 & 35.03 & 10.43 & 2.13 & 0.03 & 6.416 & 1.4 \\
\hline & $0.4-0.5$ & 0.99 & 45.22 & 33.11 & 17.64 & 3.97 & 0.07 & 6.936 & 1.5 \\
\hline & $0.5-0.6$ & 0.51 & 37.32 & 31.18 & 25.4 & 5.96 & 0.11 & 7.065 & 1.6 \\
\hline & $0.6-0.7$ & 0.05 & 32.62 & 29.22 & 30.48 & 7.50 & 0.18 & 7.657 & 1.7 \\
\hline & $0.7-0.8$ & 0.00 & 46.94 & 19.41 & 13.51 & 19.24 & 0.90 & 9.633 & 1.8 \\
\hline & $0.8-0.9$ & 0.00 & 44.99 & 20.46 & 6.53 & 26.74 & 1.28 & 9.744 & 1.9 \\
\hline & $0.9-1.0$ & 0.00 & 39.43 & 11.48 & 12.98 & 34.63 & 1.48 & 9.469 & 1.10 \\
\hline bin 2 & & 3.47 & 34.77 & 56.39 & 7.68 & 1.14 & 0.01 & 5.353 & 2 \\
\hline bin 3 & & 2.74 & 14.98 & 21.25 & 53.49 & 10.10 & 0.18 & 6.895 & 3 \\
\hline bin 4 & & 1.81 & 0.00 & 0.00 & 0.00 & 99.59 & 0.40 & 5.475 & 4 \\
\hline bin 5 & & 0.03 & 0.00 & 0.00 & 0.00 & 0.00 & 100.00 & 13.804 & 5 \\
\hline
\end{tabular}

Note: bin1 to bin5 are five size classes with corresponding effective radius.
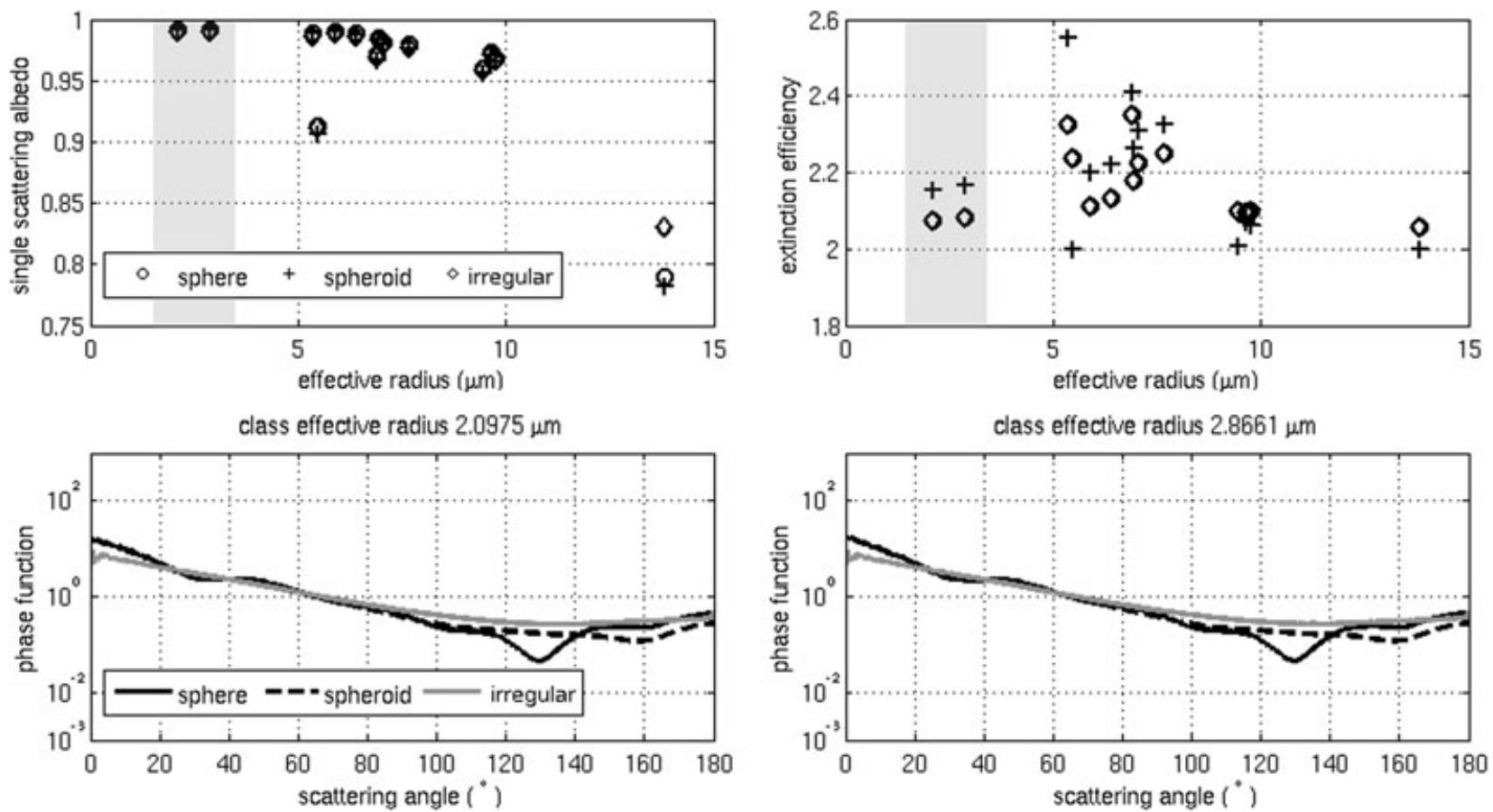

Fig. 7. Scattering properties of scattering classes for different particle shapes: (a) single scattering albedo and (b) extinction efficiency depending on the effective radius of the 14 scattering classes, shaded in grey, the two classes 1.1 and 1.2 accounting for over $80 \%$ of the dust cloud boxes; (c) and (d) show the scattering phase function for these two classes 1.1 with effective radius $2.0975 \mu \mathrm{m}$ and 1.2 with effective radius $2.8661 \mu \mathrm{m}$. 
Table 3. Scattering properties at $0.6 \mu \mathrm{m}$ (single scattering albedo [SSA], extinction efficiency [EE] of dust particles) used for Monte Carlo calculations at three wavelengths and for three different shapes (spheres [S], prolate spheroids [P], irregular particles [I])

\begin{tabular}{|c|c|c|c|c|c|c|c|c|c|c|c|c|c|c|c|c|c|c|}
\hline \multirow[b]{4}{*}{ Class } & \multicolumn{18}{|c|}{ Scattering properties of scattering classes } \\
\hline & \multicolumn{6}{|c|}{ Wavelength $0.6 \mu \mathrm{m}$} & \multicolumn{6}{|c|}{ Wavelength $0.8 \mu \mathrm{m}$} & \multicolumn{6}{|c|}{ Wavelength $1.6 \mu \mathrm{m}$} \\
\hline & \multicolumn{3}{|c|}{ SSA } & \multicolumn{3}{|c|}{$\mathrm{EE}$} & \multicolumn{3}{|c|}{ SSA } & \multicolumn{3}{|c|}{$\mathrm{EE}$} & \multicolumn{3}{|c|}{ SSA } & \multicolumn{3}{|c|}{$\mathrm{EE}$} \\
\hline & $\mathrm{S}$ & $\mathrm{P}$ & I & $\mathrm{S}$ & $\mathrm{P}$ & I & $S$ & $\mathrm{P}$ & I & $S$ & $P$ & I & $\mathrm{S}$ & $P$ & I & $S$ & $P$ & I \\
\hline 1.1 & 0.992 & 0.992 & 0.991 & .073 & 2.154 & 2.073 & 0.997 & 0.992 & 0.997 & 1.923 & 1.968 & 1.923 & 0.988 & 0.987 & 0.988 & 0.873 & 0.817 & 0.8 \\
\hline 1.2 & 0.992 & 0.992 & 0.990 & 2.081 & 2.166 & 2.081 & 0.997 & 0.992 & 0.997 & 1.945 & 1.993 & 1.945 & 0.988 & 0.987 & 0.988 & 0.893 & 0.839 & 0.893 \\
\hline 1.3 & 0.991 & 0.990 & 0.989 & 2.110 & 2.202 & 2.110 & 0.997 & 0.990 & 0.996 & 2.010 & 2.072 & 2.010 & 0.988 & 0.988 & 0.988 & 0.976 & 0.934 & 0.976 \\
\hline 1.4 & 0.988 & 0.988 & 0.987 & 2.135 & 2.220 & 2.135 & 0.996 & 0.988 & 0.996 & 2.032 & 2.117 & 2.032 & 0.988 & 0.988 & 0.988 & 1.069 & 1.056 & 1.069 \\
\hline 1.5 & 0.985 & 0.984 & 0.983 & 2.178 & 2.262 & 2.178 & 0.995 & 0.984 & 0.995 & 2.093 & 2.209 & 2.093 & 0.988 & 0.989 & 0.989 & 1.217 & 1.242 & 1.217 \\
\hline 1.6 & 0.982 & 0.979 & 0.980 & 2.226 & 2.308 & 2.226 & 0.994 & 0.979 & 0.994 & 2.164 & 2.315 & 2.164 & 0.989 & 0.989 & 0.989 & 1.380 & 1.446 & 1.380 \\
\hline 1.7 & 0.979 & 0.976 & 0.977 & 2.252 & 2.328 & 2.252 & 0.993 & 0.976 & 0.993 & 2.190 & 2.361 & 2.190 & 0.989 & 0.989 & 0.989 & 1.476 & 1.569 & 1.476 \\
\hline 1.8 & 0.973 & 0.970 & 0.972 & 2.095 & 2.069 & 2.095 & 0.991 & 0.970 & 0.991 & 1.819 & 1.858 & 1.820 & 0.984 & 0.985 & 0.985 & 1.159 & 1.178 & 1.159 \\
\hline 1.9 & 0.968 & 0.965 & 0.967 & 2.098 & 2.062 & 2.098 & 0.990 & 0.965 & 0.990 & 1.884 & 1.873 & 1.884 & 0.983 & 0.984 & 0.984 & 1.193 & 1.171 & 1.193 \\
\hline 1.10 & 0.960 & 0.956 & 0.959 & 2.098 & 2.006 & 2.098 & 0.987 & 0.956 & 0.988 & 1.790 & 1.781 & 1.790 & 0.982 & 0.983 & 0.984 & 1.298 & 1.319 & 1.298 \\
\hline 2 & 0.989 & 0.988 & 0.986 & 2.327 & 2.553 & 2.327 & 0.997 & 0.988 & 0.996 & 2.710 & 2.822 & 2.710 & 0.991 & 0.991 & 0.991 & 1.450 & 1.386 & 1.450 \\
\hline 3 & 0.972 & 0.967 & 0.969 & 2.352 & 2.410 & 2.352 & 0.991 & 0.967 & 0.991 & 2.267 & 2.543 & 2.267 & 0.989 & 0.990 & 0.990 & 1.840 & 2.060 & 1.840 \\
\hline 4 & 0.913 & 0.907 & 0.912 & 2.239 & 2.000 & 2.239 & 0.974 & 0.907 & 0.974 & 2.220 & 2.000 & 2.220 & 0.974 & 0.978 & 0.979 & 2.054 & 2.000 & 2.054 \\
\hline 5 & 0.789 & 0.783 & 0.831 & 2.058 & 2.000 & 2.058 & 0.930 & 0.783 & 0.941 & 2.125 & 2.000 & 2.125 & 0.940 & 0.938 & 0.951 & 2.231 & 2.000 & 2.231 \\
\hline
\end{tabular}

The work of Yang et al. (2007) also shows that the single scattering albedo from spheres and spheroids have only small differences, with slightly smaller values for spheroids.

There are other studies on the topic of particle shape like Kalashnikova and Sokolik (2004) dealing with mixtures of different shapes based on electron microscopy data. They show that for different shapes the differences with respect to spherical particle vary even in sign and increase with particle radius.

Fu et al. (2009) obtain the result that the single scattering albedo of spherical and spheroidal particles differ less than $1 \%$.

In Kalashnikova and Sokolik (2002) the scattering properties of mixtures of sharply edged, smooth shapes and spheres are compared. They find larger single scattering albedo for mixtures with more sharp-edged particles.

Two example phase functions of the biggest classes 1.1 and 1.2 in number concentration which are suitable for about $80 \%$ of the dust cloud boxes are presented in Fig. 7. Both classes are marked with light grey in the pictures. Most obvious is the different shape of the phase function of spheres compared to the ones for spheroids and irregular particles, especially in the region of a scattering angle between $100^{\circ}$ and $140^{\circ}$. In Yang et al. (2007) these differences can also be found although they are less pronounced.

Because the geometric optics method does not allow to calculate the extinction efficiency, it was assumed that the values for irregular particles are identical to the ones for projected area equivalent spheres. This assumption reduces the differences in the radiative fields of irregular shaped particles compared to that of spheres. Thus, all shape-induced differences in the radiation

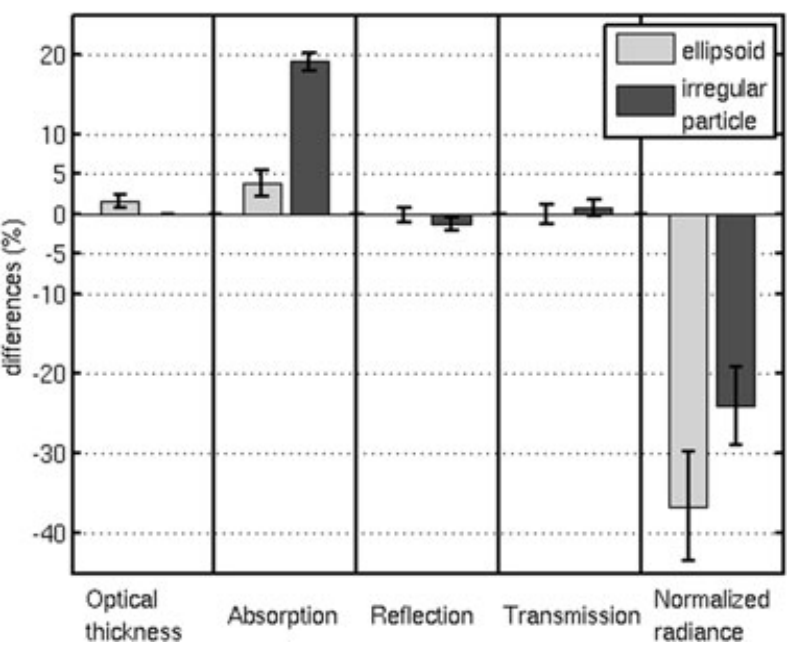

Fig. 8. Differences from simulated fields for spheres in percent to fields for spheroids and irregular particles.

fields result from the differences in single scattering albedo and scattering phase function.

In Fig. 8 the relative differences in percent in the reflectance fields for spheroids and irregular particles compared to spheres are plotted.

The optical thickness for a dust cloud consisting of irregular particles is the same as for spheres because of the assumption of identical extinction efficiencies. Large differences occur in the absorption. For spheroids the averaged absorption field is nearly $5 \%$ higher and for irregular particles it is nearly $20 \%$ higher 
than for the absorption field of spherical particles. However the absolute values are very small, so that percentaged differences might not be the best indicator for shape-induced absorption differences.

The averagesof fields of reflection and transmission differ less than $2 \%$ for irregular particles and spheroids from spheres. In Yang et al. (2007), it is shown that the reflectance of a dust cloud with spheroidal particles is much higher than the reflectance of a cloud consisting of spherical particles, which under the chosen conditions (aspect ratio and refractive index) of this study, could not be verified.

For the averaged field of normalized radiance we find lower values for spheroids and irregular particles. The normalized radiance of the dust field consisting of spheroids is about $35 \%$ lower than that with spherical dust particles. In the case of the irregular shaped particle the averaged field is about $24 \%$ lower.

These relatively large differences are caused by the different scattering phase functions. The very small differences in single scattering albedo do not have the required impact on the radiance calculations. The extinction efficiencies of spheres and irregular shaped particles do not differ, only for spheroidal particles the differences are significant. So, for the differences in the radiation fields between radiances from spherical and irregular shaped particles the main contribution comes from the differing phase functions especially in the scattering angle region that corresponds to the sun position of about $30^{\circ}$ in zenith angle and the satellites viewing geometry close to nadir.

In Fu et al. (2009), differences of less than 5\% have been described for their dust cloud reflectivity calculations.

\subsection{3-D effect}

Large-scale and high-resolution 3-D radiative transfer simulations are very expensive in computing time. A commonly used approximation for the 3-D radiative transfer is the so-called Independent Pixel Approximation (IPA), where the results of the 1-D radiative transfer calculations are added column by column to obtain the domain averaged radiative fluxes or radiances.

The only differences in 3-D and IPA is the negligence of horizontal photon transport in the IPA. In this study full 3-D and IPA calculations for a 3-D dust cloud with a sun zenith angle of $70^{\circ}$ at 31 January 2008 have been performed. The model domain shown in Fig. 9 has a width of about $1800 \mathrm{~km}$. In order to resolve 3 -D effects, the grid box size was refined from $26 \mathrm{~km}$ for the MUSCAT cloud to $6 \mathrm{~km}$ for the Monte Carlo calculations.

In Fig. 9 the simulated reflectivity field at the cloud for 1-D and 3-D calculations is shown. The field in Fig. 9(b) is calculated with only vertical photon transport (IPA) and in (c) with additional horizontal photon transport. The sun is shining from the right side of the domain. (a) shows the optical thickness of the cloud at $0.6 \mu \mathrm{m}$. The domain averaged values of the fields of normalized reflected radiance, absorption, transmission and
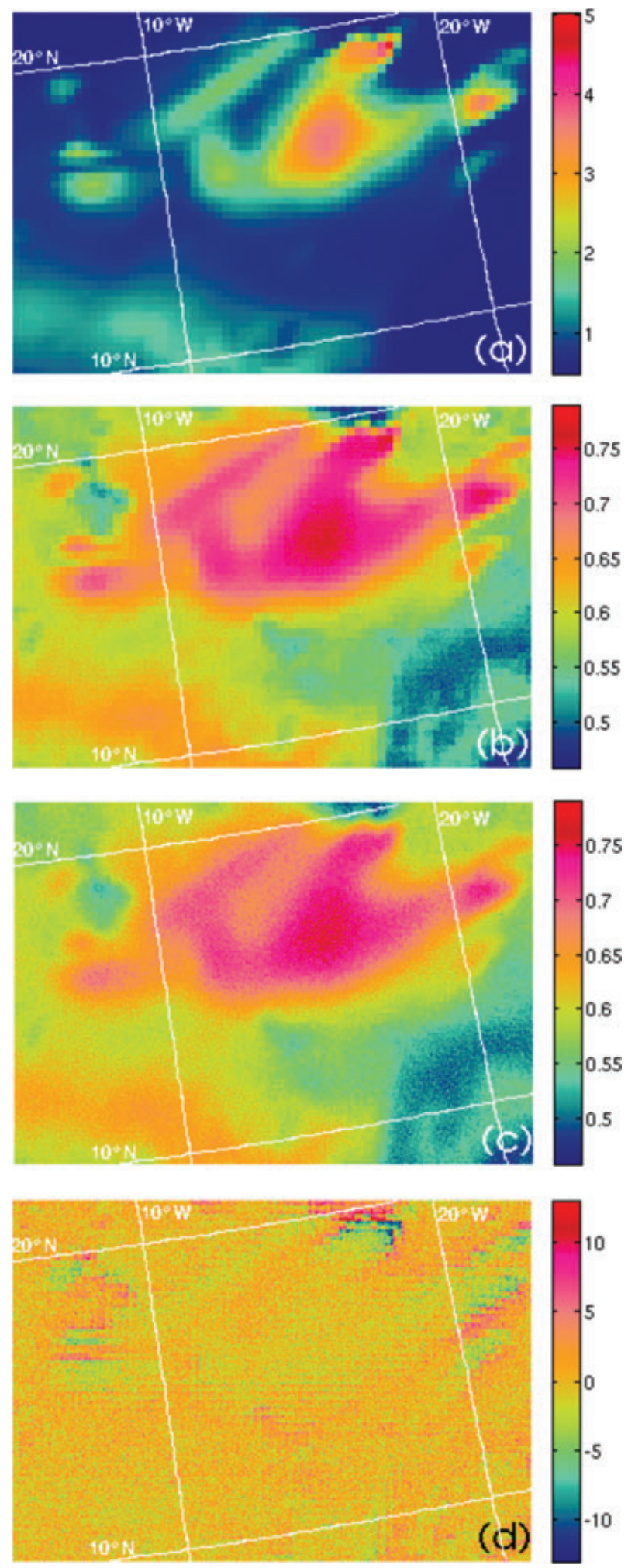

Fig. 9. Optical thickness at $0.6 \mu \mathrm{m}$ with a domain mean of 1 and a standard deviation of 0.6 (a), Monte Carlo simulated reflectance field for only vertical photon transport (b), including horizontal photon transport (c) and the differences between them in \% (d). 
reflection do not show significant differences between the 3-D and IPA 1-D calculations. Depending on the cloud structure local deviations are clearly visible. In (c) the 'edges' in the dust cloud are smoothed out due to the horizontal photon transport. Picture (d) shows the differences between both reflection fields. Where the gradients in optical thickness of the dust cloud are largest, the 3-D effect is most pronounced. In some areas the radiance fields for the 3 -D scenario differ about $\pm 15 \%$ from the fields for the 1-D scenario.

The aspect of 1-D and 3-D photon transport in radiative transfer simulations for water clouds in the atmosphere is discussed in Benner and Evans (2001). Their results fit well to ours for dust clouds. The average radiative fields do not differ significantly, but depending on the cloud structure there are big differences in local cloud areas.

Davis et al. (1997) have also shown the smoothing effect of horizontal photon transport in cloudy atmosphere.

Di Giuseppe and Tompkins (2003) show that the effect of horizontal photon transport is negligible for the mean quantities of reflection, transmission and absorption, but for high variability in water cloud structure the effect increases. Also the dependence on the sun zenith angle is considered.

The comparison for I3RC with 3-D and 1-D Monte Carlo models shows that the 3-D effect is larger for lower sun zenith angle. For the step cloud with high gradient in optical thickness, the 3-D effect is most pronounced (Cahalan et al., 2005).

\section{Summary and conclusions}

By means of a Monte Carlo radiative transfer model the full 3-D radiative transfer study has been performed where extinction, scattering and absorption are subject to spatial variability. The spatial dust cloud under investigation has been derived from a mesoscale cloud resolving atmospheric circulation model with a microphysical dust scheme. The effect of different assumptions on dust particle geometries on the reflected, transmitted and absorbed radiation fluxes as well as on the horizontal reflectance field has been investigated. Significant differences in radiative properties have been found for spherical, spheroidal and irregular shaped particles. 3-D radiative transfer effects are negligible except near the edges of dust clouds. It is obvious that dust particles are neither spherical nor of spheroidal shape. However, the consideration of a more realistic irregular particle geometry remains difficult due to the lack of available light scattering theories. The results from the geometric optics approximation used in this study can only be regarded as a hint towards the importance of dust cloud microphysics on their radiative effects and for dust remote sensing from satellite.

\section{Acknowledgments}

The SAMUM research group was funded by the Deutsche Forschungsgemeinschaft (DFG) under grant number FOR 539.
The authors thank the German Aerospace Center for partial support of this work through contract 23210572.

\section{References}

Ansmann, A., Petzold, A., Kandler, K., Tegen, I., Wendisch, M. and co-authors. 2011. Saharan Mineral Dust Experiments SAMUM 1 and SAMUM 2: what have we learned? Tellus 63B, this issue.

Benner, T. C. and Evans, K. F. 2001. Three-dimensional solar radiative transfer in small tropical cumulus fields derived from high-resolution imagery. J. Geophys. Res. 106, 14975-14984.

Bristow, C. S., Drake, N. and Armitage, S. 2009. Deflation in the dustiest place on Earth: The Bodele Depression, Chad. In: Geomorphology, Contemporary research in aeolian geomorphology, 6th International Conference on Aeolian Research (ICAR VI) 105(1-2), University of Guelph, Ontario, Canada, pp. 50-58.

Bristow, C. S., Hudson-Edwards, K. A. and Chappell, A. 2010. Fertilizing the Amazon and equatorial Atlantic with West African dust. Geophys. Res. Lett. 37, 1-5.

Cahalan, R. F., Oreopoulos, L., Marshak, A., Evans, K., Davis, A. B., and co-authors. 2005. THE I3RC: bringing together the most advanced radiative transfer tools for cloudy atmospheres. Bull. Amer. Meteor. Soc. 86, 1275-1293.

Davis, A., Marshak, A., Cahalan, R. F. and Wiscombe, W. 1997. The Landsat scale break in stratocumulus as a three-dimensional radiative transfer effect: implications for cloud remote sensing. J. Atmos. Sci. 54, 241-260.

Di Giuseppe, F. and Tompkins, A. M. 2003. Effect of spatial organisation on solar radiative transfer in three dimensional idealised stratocumulus cloud fields. J. Atmos. Sci. 60, 1774-1794.

Ernst, T., Rother, T., Schreier, F., Wauer, J. and Balzer, W. 2003. DLR's VirtualLab: Scientific Software Just a Mouse Click Away Comput. Sci. Eng. 5(1), 70-79, doi:10.1109/MCISE.2003.1166555.

Evan, A. T., Dunion, J., Foley, J. A., Heidinger, A. K. and Velden, C. S. 2006. New evidence for a relationship between Atlantic tropical cyclone activity and African dust outbreaks. Geophys. Res. Lett. 33, $1-5$.

Fu, Q., Thorsen, T. J., Su, J., Ge, J. and Huang, J. 2009. Test of Mie-based single-scattering properties of non-spherical dust aerosols in radiative flux calculations. J. Quant. Spectroscopy \& Radiative Transf. 110(1416), 1640-1653, doi:10.1016/j.jqsrt.2009.03.01.

Garrison, V. H., Shinn, E. A., Foreman, W. T., Griffin, D. W., Holmes, and co-authors. 2003. African and Asian dust: from desert soils to coral reefs. BioScience 53, 469-480.

Griffin, D. W., Garrison, V. H., Kellogg, C. and Shinn, E. A. 2001. African desert dust in the Caribbean atmosphere: microbiology and public health. Aerobiologia 17, 203-213.

Heinold, B., Helmert, J., Hellmuth, O., Wolke, R., Ansmann, A. and co-authors. 2007. Regional Modeling of Saharan Dust Events using LM-MUSCAT: model description and case studies. J. Geophys. Res. 112, 21 PP., D11204, doi:10.1029/2006JD007443.

Heintzenberg, J. 2009. The SAMUM-1 experiment over Southern Morocco: overview and introduction. Tellus 61B, 2-11.

Kalashnikova, O. V. and Sokolik, I. N. 2002. Importance of shapes and compositions of wind-blown dust particles for remote sensing at solar wavelengths. Geophys. Res. Lett. 29(10) 1-4. 
Kalashnikova, O. V. and Sokolik, I. N. 2004. Modeling the radiative properties of nonspherical soil-derived mineral aerosols. J. Quant. Spectroscopy \&Radiative Transf. 87(2), 137-166.

Kandler, K., Schütz, L., Deutscher, C., Ebert, M., Hofmann and coauthors. 2009. Size distribution, mass concentration, chemical and mineralogical composition and derived optical parameters of the boundary layer aerosol at Tinfou, Morocco, during SAMUM 2006. Tellus 61B, 32-50.

Kandler, K., Lieke, K., Benker, N., Emmel, C., Küpper, M. and coauthors. 2011. Electron microscopy of particles collected at Praia, Cape Verde, during the Saharan Mineral dust experiment: particle chemistry, shape, mixing state and complex refractive index. Tellus 63B, this issue.

Kaufman, Y. J., Koren, I., Remer, L. A., Rosenfeld, D. and Rudich, Y. 2005. The effect of smoke, dust, and pollution aerosol on shallow cloud development over the Atlantic Ocean. In: Proceedings of the National Academy of Sciences of the United States of America, 102, no.32, 11 207-11 212.

Macke, A. 1993. Scattering of light by polyhedral ice crystals. Appl. Opt. 32, 2780-2788.

Macke, A., Mueller, J. and Raschke, E. 1996. Single scattering properties of atmospheric ice crystals. J. Atmos. Sci. 53, 2813-2825.

Macke, A., Mishchenko, M. -I., Carlson, B. -E. and Muinonen, K. 1997. Scattering of light by large spherical, spheroidal, and circular cylindrical scatterers: geometrical optics approximation versus T-matrix method. In: IRS 96: Current Problems in Atmospheric Radiation. (eds) Smith W-L, Stamnes K. A. Deepak Publ., Hampton., 822-825.

Macke, A., Mitchell, D. L. and Bremen, L. V. 1999. Monte Carlo radiative transfer calculations for inhomogeneous mixed phase clouds. Phys. Chem. Earth, Part B: Hydrol. Oceans Atmos. 24, 3, 237-241.

Marshak, A., Davis, A. (Eds.), 2005. 3D radiative transfer in cloudy atmospheres. In: Series: Physics of Earth and Space Environments, XII, Springer, Berlin, 686 p., ISBN 978-3-540-23958-1.
Otto, S., Bierwirth, E., Weinzierl, B., Kandler, K., Esselborn, M. and coauthors. 2009. Solar radiative effects of a Saharan dust plume observed during SAMUM assuming spheroidal model particles. Tellus 61B, 270-296.

Scheirer, R. and Macke, A. 2003. Cloud inhomogeneity and broadband solar fluxes. J. Geophys. Res. 108(D19), 1-8.

Schlimme, I., Macke, A. and Reichardt, J. 2005. The impact of ice crystal shapes, size distributions, and spatial structures of sirrus clouds on solar radiative fluxes. J. Atmos. Sci. 62(7), 2274-2283.

Schepanski, K., Tegen, I., Laurent, B., Heinold, B. and Macke, A. 2007. A new Saharan dust source activation frequency map derived from MSG-SEVIRI IR-channels. Geophys. Res. Lett. 34, 1-5.

Schmidt, K., Wauer, J., Rother, T. and Trautmann, T. 2009. Scattering database for spheroidal particles. Appl. Opt. 48, 2154-2164.

Tegen, I. and Lacis, A. A. 1996. Modeling of particle size distribution and its influence on the radiative properties of mineral dust aerosol. J. Geophys. Res. 101(D14), 19237-19244.

Tegen, I., Lacis, A. A. and Fung, I. 1996. The influence on climate forcing of mineral aerosols from disturbed soils. Nature 380, 419-422.

Tegen, I., Harrison, S. P., Kohfeld, K. E., Prentice, I. C., Coe, M. and co-authors. 2002. Impact of vegetation and preferential source areas on global dust aerosol: results from a model study. J. Geophys. Res. 107, 1-21.

Wald, A. E., Kaufman, Y. J., Tanr, D. and Gao, B.-C. 1998. Daytime and nighttime detection of mineral dust over desert using infrared spectral contrast. J. Geophys. Res. 103(D24), 32 307-32 313.

Wauer, J., Schmidt, K., Rother, T., Ernst, T. and Hess, M. 2004. Two software tools for plane wave scattering on nonspherical particles in the German Aerospace Center's virtual laboratory Appl. Opt. 43, 6371-6379.

Yang, P., Feng, Q., Hong, G., Kattawar, G.W., Wiscombe, W. J. and co-authors. 2007. Modeling of the scattering and radiative properties of nonspherical dust particles. J. Aerosol Sci. 38, 995-1014. 